\title{
Resonant Behavior in a Periodically Forced Non-Isothermal Oregonator
}

David García-Selfa ${ }^{a}$, Alberto P. Muñuzuria ${ }^{a, b}$, Juan Pérez-Mercader ${ }^{b, c}$ and David S. A. Simakov*,d

${ }^{a}$ Group of Nonlinear Physics, Universidad de Santiago de Compostela, Campus Sur, 15782 Santiago de Compostela, Spain

${ }^{b}$ Department of Earth and Planetary Sciences, Origins of Life Initiative, Harvard University, Cambridge, Massachusetts 02138, United States

c Santa Fe Institute, Santa Fe, New Mexico 87501, United States

${ }^{d}$ Department of Chemical Engineering, University of Waterloo, Waterloo, Ontario N2L 3G1, Canada

\section{Supporting Information}

\section{Non-isothermal Oregonator model equations}

The energy balance for a continuous stirred tank reactor (CSTR) is given as follows:

$$
V_{R} \rho_{w} C_{p w} \frac{d T}{d t}=-V_{R} \sum_{i} \Delta H_{R i} r_{i}+Q_{f} \sum_{j} C_{j f}\left(H_{j f}-H_{j}\right)+\dot{Q}+V_{R} R_{I R}
$$

- $V_{R}-$ reactor volume

- $\quad \rho_{w}$ - fluid (water) density

- $C_{p w}-$ constant-pressure heat capacity (of water)

- $\Delta H_{R i}$ - enthalpy change for reaction $i ; \Delta H_{R i}=\Delta H_{R i}^{0}+\int_{T_{r}}^{T} \Delta C_{p i} d T \approx \Delta H_{R i}^{0}\left(\Delta C_{p i} \approx 0\right)$

- $\quad r_{i}$ - reaction rate for reaction $i$

- $Q_{f}$ - volumetric feed flow rate

- $\quad C_{j f}-$ feed concentration of specie $j$

- $H_{j}-$ specific enthalpy of specie $j$

- $\quad \dot{Q}$ - heat transfer rate; $\dot{Q}=U S\left(T_{c}-T\right)$

- $S$ - heat transfer surface

- $T_{c}-$ coolant temperature 
- $U$-wall heat transfer coefficient

- $R_{I R}$ - infrared irradiation heating rate (per reactor volume)

Reaction rates are given as follows:

$r_{1}(T)=k_{1} A Y H^{2}, r_{2}(T)=k_{2} X Y H, r_{3}(T)=k_{2} A X, r_{4}(T)=k_{4} X^{2}, r_{5}(T)=k_{5} B Z$

$k_{i}(T)=k_{i 0} \exp \left[-\frac{E_{i}}{R}\left(\frac{1}{T}-\frac{1}{T_{0}}\right)\right], k_{i 0}=k_{i}\left(T_{0}\right)$

Assuming that the term $Q_{f} \sum_{j} C_{j f}\left(H_{j f}-H_{j}\right)$ in the energy balance is negligible and after substituting reaction rates and the heat transfer term:

$$
\begin{aligned}
V_{R} \rho_{w} C_{p w} \frac{d T}{d t} & =-V_{R}\left(k_{1} A Y H^{2} \Delta H_{R 1}+k_{2} X Y H \Delta H_{R 2}+k_{3} A X H \Delta H_{R 3}+k_{4} X^{2} \Delta H_{R 4}+k_{5} B Z \Delta H_{R 5}\right) \\
& +U S\left(T_{c}-T\right)+V_{R} R_{I R}
\end{aligned}
$$

Dimensionless concentrations, time and temperature are defined as follows:

$$
x=\frac{2 k_{4}}{k_{3} A H} X, \quad y=\frac{k_{2}}{k_{3} A} Y, z=\frac{k_{4} k_{5} B}{\left(k_{3} A H\right)^{2}} Z, \tau=k_{5} B t, \theta=\frac{T}{T_{c}}
$$

After some algebraic manipulations, the following dimensionless energy balance is obtained:

$$
\gamma \frac{d \theta}{d \tau}=\psi(x, y, z, \theta)+(1-\theta)+\omega
$$

- $\gamma(\theta)=\frac{V_{R} \rho_{w} C_{p w} k_{5} B}{U S}$

- $\psi(x, y, z, \theta)=-\frac{V_{R}}{U S T_{c}}\left(\alpha_{1} y+\alpha_{2} x y+\alpha_{3} x+\alpha_{4} x^{2}+\alpha_{5} z\right)$
○ $\alpha_{1}(\theta)=\frac{k_{1} k_{3} A^{2} H^{2}}{k_{2}} \Delta H_{R 1}$
○ $\alpha_{2}(\theta)=\frac{k_{3}^{2} A^{2} H^{2}}{2 k_{4}} \Delta H_{R 2}$
○ $\alpha_{3}(\theta)=\frac{k_{3}^{2} A^{2} H^{2}}{2 k_{4}} \Delta H_{R 3}$
○ $\alpha_{4}(\theta)=\frac{k_{3}^{2} A^{2} H^{2}}{4 k_{4}} \Delta H_{R 4}$ 
$\begin{aligned} & \circ \quad \alpha_{5}(\theta)=\frac{k_{3}^{2} A^{2} H^{2}}{k_{4}} \Delta H_{R 5} \\ \text { - } \omega & =\frac{V_{R} R_{I R}}{U S T_{c}}\end{aligned}$

\section{Values of the parameters used in simulations}

Values below were used in all simulations (other values used for specific simulations are given in the main text):

- Initial values in simulations: $x_{0}=0, y_{0}=0.1, z_{0}=0, \theta_{0}=1$

- Gas constant and reference temperature: $R=8.314 \mathrm{~J} /(\mathrm{mol} \mathrm{K}), T_{0}=298 \mathrm{~K}$

- Reactor geometry and fluid properties: $V_{R}=0.05 \mathrm{~L}, S=1 \mathrm{dm}^{2}, \rho_{w}=1 \mathrm{~kg} / \mathrm{L}, C_{p w}$ $=4.186 \mathrm{~kJ} /(\mathrm{kg} \mathrm{K})$

- Reaction kinetics parameters: $A_{0}=0.24 \mathrm{M}, B_{0}=0.18 \mathrm{M}, f=1, k_{f}=5.556 \times 10^{-4} \mathrm{~s}^{-1}$

- Reaction enthalpies, activation energies and rate constants (Pullela et al., Phys. Chem. Chem. Phys. 2009):

\begin{tabular}{|c||c|c|c|c|c|}
\hline Reaction: & 1 & 2 & 3 & 4 & 5 \\
\hline$\Delta H_{R i}\left[\mathrm{~kJ} \mathrm{~mol}^{-1}\right]$ & 43 & -71 & 98 & -114 & 0 \\
\hline$E_{i}\left[\mathrm{~kJ} \mathrm{~mol}^{-1}\right]$ & 60 & 25 & 60 & 75 & 70 \\
\hline$k_{i 0}$ & $2\left[\mathrm{M}^{-3} \mathrm{~s}^{-1}\right]$ & $10^{6}\left[\mathrm{M}^{-2} \mathrm{~s}^{-1}\right]$ & $10\left[\mathrm{M}^{-2} \mathrm{~s}^{-1}\right]$ & $2 \times 10^{3}\left[\mathrm{M}^{-1} \mathrm{~s}^{-1}\right]$ & $1\left[\mathrm{M}^{-1} \mathrm{~s}^{-1}\right]$ \\
\hline
\end{tabular}




\section{Simulation results}
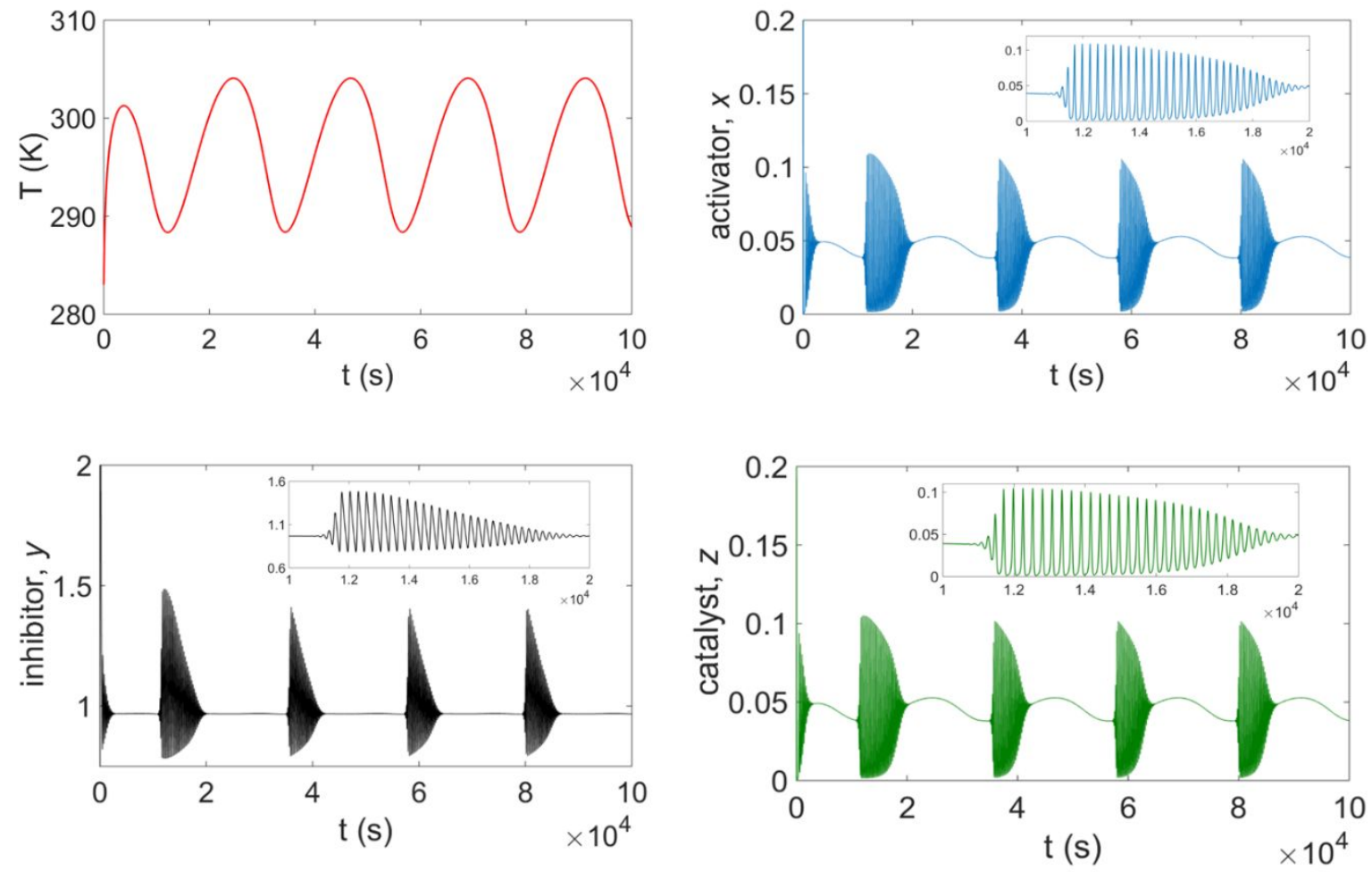

Figure S1. System response to the periodic (sinusoidal) forcing at $\mathrm{f}_{\mathrm{f}}=4.5 \times 10^{-5} \mathrm{~Hz}$, showing the temporal evolution of the reactor temperature and dimensionless concentrations of the activator, inhibitor and catalyst. Parameters: $H_{0}=0.1 \mathrm{M}, T_{c}=283 \mathrm{~K}, U=10 \mathrm{~J} \mathrm{~m}^{-2} \mathrm{~K}^{-1} \mathrm{~s}^{-1}, \boldsymbol{R}_{\boldsymbol{I R} \boldsymbol{\theta}}=\mathbf{0 . 0 2 8} \mathbf{~ k W} / \mathbf{L}, A_{f}=0.018 \mathrm{~kW} / \mathrm{L}$.
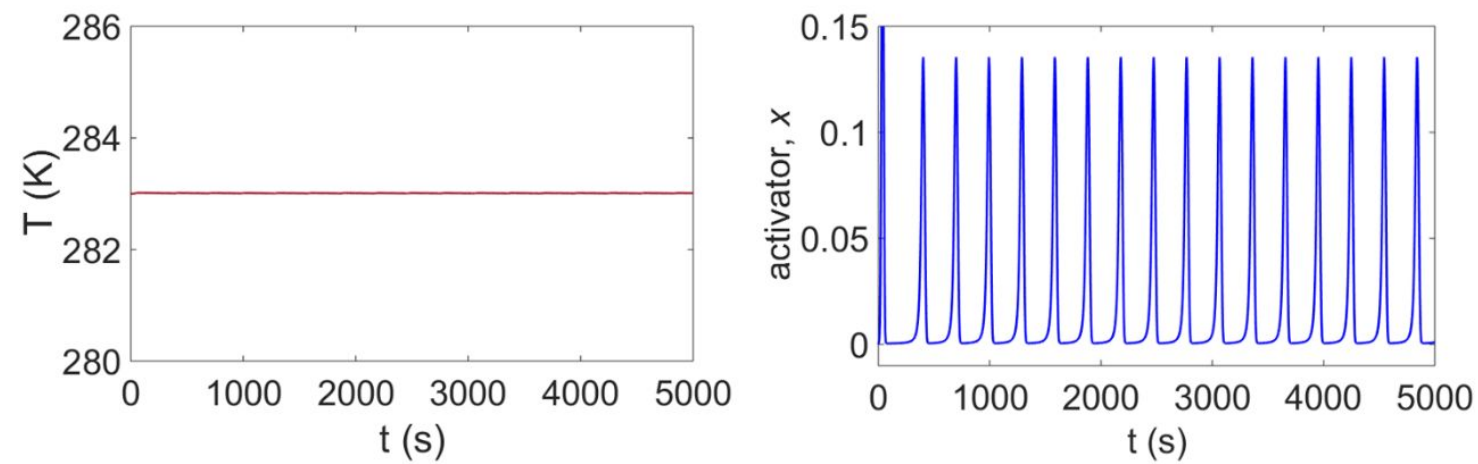

Figure S2. Self-oscillations in the absence of external forcing, showing the temporal evolution of the reactor temperature and dimensionless activator concentration. Parameters: $H_{0}=0.1 \mathrm{M}, T_{c}=283 \mathrm{~K}, U$ $=10 \mathrm{~J} \mathrm{~m}^{-2} \mathrm{~K}^{-1} \mathrm{~s}^{-1}, \boldsymbol{R}_{\boldsymbol{I R} \boldsymbol{0}}=\mathbf{0} \mathbf{k W} / \mathbf{L}$. 

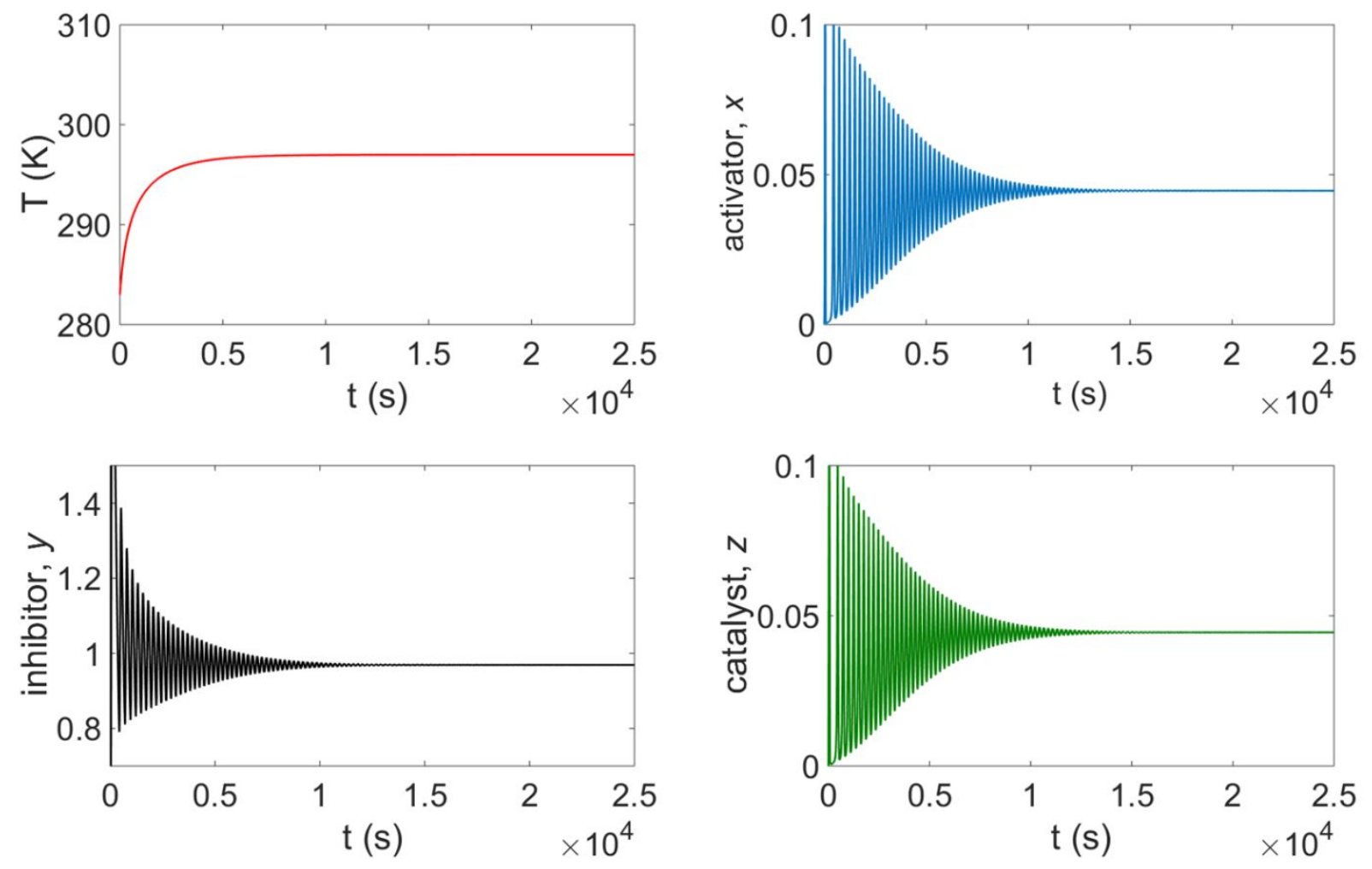

Figure S3. The case of constant external forcing, showing the temporal evolution of the reactor temperature and dimensionless concentrations of the activator, inhibitor and catalyst. Parameters: $H_{0}=$ $0.1 \mathrm{M}, T_{c}=283 \mathrm{~K}, U=10 \mathrm{~J} \mathrm{~m}^{-2} \mathrm{~K}^{-1} \mathrm{~s}^{-1}, \boldsymbol{R}_{I R \boldsymbol{\theta}}=0.028 \mathrm{~kW} / \mathbf{L}$. 